\title{
INTEGRAÇÃO ENSINO-SERVIÇOS DE SAÚDE: O INTERNATO RURAL MÉDICO DA UNIVERSIDADE FEDERAL DE MATO GROSSO
}

\author{
TEACHING-HEALTH SERVICE INTEGRATION: THE RURAL MEDICAL BOARDING SCHOOL OF THE \\ FEDERAL UNIVERSITY OF MATO GROSSO
}

Marco Aurelio Bertúlio das Neves ${ }^{1}$

Maria Angelica Spinelli ${ }^{2}$

Resumo Este estudo é uma pesquisa avaliativa que reconstitui e analisa os objetivos do programa de integração ensino-serviços de saúde, instituído entre a Universidade Federal de Mato Grosso, Secretaria de Estado de Saúde de Mato Grosso e o Consórcio Intermunicipal de Saúde da Região do Teles Pires, em Mato Grosso, no ano de 2000. É um estudo de caso que utilizou abordagem qualitativa e quantitativa. A coleta de dados se deu em fontes secundárias e primárias por meio de entrevistas semi-estruturadas com gestores e agentes implementadores das instituições. Os resultados demonstram, entre outros aspectos, que: não houve formulação prévia e comum dos objetivos para o programa entre as instituições; ao serem reconstituídos, verificou-se que foram elevados os percentuais de objetivos não alcançados; as atividades desenvolvidas não foram suficientes para o alcance dos objetivos; mesmo entre os atores de uma mesma instituição, os objetivos foram distintos; dos objetivos reconstituídos, $75 \%$ foram objetivos de organização de serviços ou objetivos acadêmicos. O estudo conclui que as instituições visaram seus objetivos específicos. Recomenda o estabelecimento de referencial teórico sobre integração ensino-serviços de saúde que paute a definição de objetivos, metas, atividades, responsabilidades e indicadores para futuras avaliações sistemáticas do programa.

Palavras-chave integração ensino-serviços de saúde; pesquisa avaliativa; educação médica.
Abstract This study is an assessment survey that reconstitutes and analyzes the objectives of the teaching-health service integration program instituted by the Federal University of Mato Grosso (UFMT), by the Mato Grosso State Health Department, and by the Inter-municipal Consortium of Health of the Teles Pires Region (Mato Grosso), in 2000. It is a case study based the qualitative and quantitative approach. Data were collected from secondary and primary sources by means of semi-structured interviews conducted with managers and agents who implemented the institutions. The results show, among other aspects, that there was no previous and common formulation of program objectives among the institutions; when reconstituted, it was noticed that the index of unachieved objectives was very high; the activities that were carried out were insufficient to reach the objectives; even among the players of a same institution, the objectives were distinct; of the reconstituted objectives, $75 \%$ were service organization or academic objectives. The study concludes that each institution sought its specific objectives and recommends the establishment of a theoretical referential on the teaching-health service integration process to guide the definition of the objectives, goals, activities, responsibilities, and indicators for future systematic program assessments.

Keywords teaching-health service integration; evaluative research; medical education. 


\section{Introdução}

A formação dos profissionais de saúde, em especial do profissional médico, vem sofrendo nas últimas três décadas na América Latina e, em particular, no Brasil de problemas diretamente ligados às estratégias de ensino e na adequação desses profissionais à realidade dos sistemas de saúde. A necessidade de mudança na gestão e na formação de recursos humanos tem sido amplamente reconhecida nos últimos anos, enfocando, principalmente a graduação médica, quando realizada em integração entre os centros formadores e os serviços de saúde.

Distintas estratégias foram utilizadas em busca de mudanças na educação médica no Brasil. Para Lampert (2002), destacam-se a Network of Community-Oriented Educational Institutions for Health Sciences, Changing Medical Education, Projeto Uma Nova Iniciativa na Educação dos Profissionais de Saúde: União com a Comunidade (UNI), Comissão Interinstitucional Nacional de Avaliação das Escolas Médicas (Cinaem) e a Gestão de Qualidade.

A proposta de integração entre as universidades e os serviços de saúde surge com o intuito de equilibrar a qualidade e quantidade de profissionais que deveriam ser formados e de integrar o ensino médico a diversas instâncias dos serviços de saúde. As primeiras iniciativas que utilizaram essa estratégia situam-se nos marcos da Integração Docente-Assistencial ${ }^{3}$ (IDA). Essa proposta de integração defendeu um novo processo de ensino-aprendizagem, mediante vivências de práticas na realidade dos serviços de saúde por discentes e docentes, maior participação dos discentes nas discussões acerca do currículo e conteúdos acadêmicos, e interação permanente entre ensino e serviços (Marsiglia, 1995).

Segundo Rodrigues (1990), as principais tendências de reorientação da educação médica na América Latina nos anos 70 e 80 foram: a) redefinição do objeto de estudo, representada pela substituição de práticas educativas organizadas em torno de um corpo de conhecimentos centrados nos objetivos de cada uma das disciplinas por processos amplos que abordassem conhecimentos referidos à saúde da população, nos marcos de uma concepção de processo saúde-doença; b) desenvolvimento de enfoques interdisciplinares; c) desenvolvimento da integração docente assistencial, concebida como um verdadeiro processo de serviço à comunidade, no qual as ações de saúde deveriam ser vistas como objetos de pesquisa, geradoras de novos conhecimentos e de novas práticas de atenção; d) incorporação e reconceituação das ciências sociais nos processos educacionais; e e) inserção da universidade, de forma crítica, nos processos de transformação dos sistemas de saúde. 
Considerando os resultados das diversas avaliações das experiências IDA ocorridas na América Latina, na década de 1980 e o acompanhamento de iniciativa similar, denominada "Community Partnerships with Health Professions Education", a Coordenação de Programas para a América Latina da Fundação Kellogg deu início, em 1990, a um novo Programa Integração Ensino Serviços de Saúde (Piess) (Almeida, 1999b). Esse programa recebeu o nome de "Uma Nova Iniciativa na Educação dos Profissionais de Saúde: União com a Comunidade" (UNI) e visava à maior articulação entre o ensino das várias profissões de saúde e à incorporação da comunidade nos esforços de colaboração entre as instituições educacionais e de serviço. Como concepção teórico-metodológica a proposta UNI contemplou três componentes: universidade, serviços de saúde e comunidade. Tinha como objetivo produzir mudanças na educação dos profissionais de saúde, na organização e no funcionamento dos serviços de saúde e na participação da comunidade nas decisões que interferem em sua qualidade de vida (Kisil, 1996).

Proveniente do processo de amadurecimento, os movimentos de IDA e UNI no Brasil se integraram, formando, em julho de 1996, a Rede Unida. A Rede é entendida como um espaço de troca e divulgação de experiências de articulação entre universidade, instituições de ensino e pesquisa, serviços, comunidade e tem como objetivo promover mudanças no modelo de atenção, no modelo de ensino em saúde e nas formas de participação social, coerentes com os princípios do Sistema Único de Saúde (SUS). Conformou-se como um ator político, no sentido de intervir sobre a formulação e execução de políticas públicas de saúde e de educação, com os propósitos de mudança pactuados entre seus pares.

A Rede tem experiências acumuladas na Argentina, Bolívia, Chile, Colômbia, Equador, México, Nicarágua, Peru, Uruguai, Venezuela e, no Brasil, com projetos na Bahia, Botucatu, Londrina, Marília e Natal.

Muitos desses projetos atestam a complexidade do trabalho articulado entre os seus componentes. Ao analisar os do Brasil, percebe-se ter sido fundamental os participantes reconhecerem, de início, suas diferenças e se conscientizarem da inexistência de fórmulas mágicas para a resolução dos conflitos. Na quase totalidade desses projetos, percebeu-se a necessidade de: identificação de elementos de interesse comum para o trabalho conjunto; a gerência reconhecer essas alianças como mutantes; envolvimento dos participantes desde o início do processo; e reconhecimento da realidade como eixo articulador do ensino, da pesquisa e da prestação de serviços.

O projeto UNI Natal é um exemplo de efetiva articulação entre os componentes propostos pela Rede. Envolve a Universidade Federal do Rio Grande do Norte, Secretaria Estadual de Saúde Pública, Secretaria 
Municipal de Saúde e Comunidade. Foram estabelecidos objetivos claros e comuns, bem como responsabilidades para as instituições envolvidas.

Já o projeto UNI Botucatu, em atividade desde 1992, tem funcionado como elemento catalisador e aglutinador de diversos processos de transformação, merecendo destaque a Reforma Curricular no Curso de Medicina, com reformulação de objetivos, métodos e conteúdos, deslocando-se, mesmo que parcialmente, para serviços básicos a hospitais comunitários. Para Machado (1999), essa experiência apresenta avanços concretos no ensino, na assistência à saúde e na participação comunitária.

Desde 1986, na Universidade Federal de Mato Grosso (UFMT), uma das maneiras como a integração com os Serviços de Saúde vem ocorrendo, tem sido através da realização dos estágios em Saúde Coletiva I e II do Curso de Medicina, na capital e em municípios do interior do estado. O estágio em Saúde Coletiva II (Internato Rural Médico) compõe o conjunto das disciplinas do sexto ano do curso de Medicina da Faculdade de Ciências Médicas (FCM) da UFMT e consiste em momento teórico-prático de aprendizado dos alunos.

Em análise do desenvolvimento dos recursos humanos em saúde nos últimos dez anos, Belaciano (1996) apontou questões relevantes para o insucesso dessa área, merecendo destaque: a) o desconhecimento e descompasso da realidade dos serviços de saúde por parte das universidades; b) as bases muito frágeis de relacionamento entre o setor educacional e de saúde; c) o descompromisso ético, humano e social dos docentes, discentes e profissionais com os usuários dos serviços; d) a desconsideração do trabalho como princípio pedagógico (a íntima relação entre o que se pratica e o que se aprende); e) a compartimentalização do ensino, os conteúdos redundantes, repetitivos e muitas vezes irrelevantes para o exercício profissional; f) a inadequação da formação frente às necessidades dos serviços de saúde; g) o uso dos serviços e da realidade social como fonte de dados para pesquisa de uso exclusivo; e h) o descompromisso da universidade com a busca de um novo modelo assistencial.

Para Lampert (2002), a diversificação de cenários de ensino e a introdução de novas práticas de saúde, apesar de desempenhar papel fundamental na mudança do perfil do médico formado, acabaram por criar novos problemas. Nessa tentativa, ocorreram relações unilaterais e de subjugação; a universidade privilegiou os serviços para atividades do seu interesse, sem considerar as necessidades das atividades de atendimento, criando demandas que não puderam ser sustentadas.

Por entender que alguns desses pontos são pertinentes à formação dos recursos humanos em saúde em Mato Grosso, e considerando a proposta de reorganização desses serviços através da estratégia da Saúde da Família no País, acredita-se que este estudo possibilitará a reflexão no interior da 
UFMT e dos serviços de saúde acerca das estratégias utilizadas para a integração entre o ensino e esses serviços, bem como proporcionará informações acerca do processo 4 e das relações 5 de ensino.

Os questionamentos formulados quando do início da pesquisa podem ser assim resumidos: como implementar a integração entre os serviços de saúde e a academia? Seria a realização do Internato Rural Médico em municípios pertencentes à área de um Consórcio Intermunicipal de Saúde uma estratégia viável para construir relações cooperativas que contribuíssem para a melhoria dos serviços de saúde e da formação médica em Mato Grosso? A integração formalizada através de convênio seria uma estratégia suficiente para o alcance dos objetivos das instituições envolvidas?

Apesar desses questionamentos e da pesquisa avaliativa se mostrar mais adequada ao estudo, a premissa teórica que referencia a avaliação por objetivos não poderia ser atendida, uma vez que a hipótese do estudo baseou-se na integração entre a UFMT, Secretaria de Estado de Saúde de Mato Grosso (SES-MT) e o Consórcio Intermunicipal de Saúde da Região do Teles Pires (CISRTP-MT), cujos objetivos não foram previamente definidos de forma clara e consensual. Assim, o êxito do programa não poderia ser determinado em função de medidas que expressassem o alcance dos objetivos do programa.

Assim, direcionou-se o estudo para o processo de formulação dos objetivos do programa, utilizando o suporte teórico utilizado por Ballart (1992), no estudo do caso do Plan Integral de Roquetes, que propõe um desenho de pesquisa avaliativa específica para os casos, em que os objetivos não estão previamente definidos, claros e consensuados entre os pares.

Esse estudo 6 teve como metas: reconstituir e analisar os objetivos da integração entre a UFMT, SES-MT e o CISRTP/MT, que regeram a realização do Internato Rural Médico do curso de Medicina no ano de 2000; identificar as atividades desenvolvidas e analisar a pertinência destas para o alcance dos objetivos reconstituídos; e identificar o grau de coincidência entre os objetivos dos gestores institucionais, agentes implementadores.

\section{Fundamentação teórica}

A pesquisa avaliativa é um processo guiado teoricamente, seguindo uma sistematização para a obtenção dos dados, com o intuito de garantir o rigor necessário para um estudo desta natureza. É um juízo de valor, um julgamento fundamentado sobre uma ação social realizada por pessoas, grupos de trabalho ou instituições (Martinic, 1997). 
Para Denis e Champagne (2000), essa pesquisa é um processo contínuo de aprendizagem, pois associa o saber obtido nas conclusões às recomendações para a melhoria da qualidade das intervenções.

Apesar da diversidade de conceitos de avaliação presentes na literatura e de certa semelhança entre eles, o conceito que mais se aplica a este estudo é o apresentado por Martinic, o qual considera avaliação como: "um processo onde a coleta sistemática de dados permite emitir um julgamento, um valor sobre uma política ou um programa, e os resultados servem para subsidiar o planejamento e a tomada de decisão" (Martinic, 1997, p. 45).

Diante da diversidade de classificações existentes referentes às formas de avaliação, adotamos como referência a de Aguilar e Ander-Egg (1994), ou seja, segundo o momento em que se avalia a procedência do avaliador e a função da avaliação.

Segundo o momento em que se avalia, esta avaliação pode ser ex ante, durante e ex post. As avaliações ex ante são feitas para auxiliar na tomada da decisão de empreender ou não o programa; consiste em avaliar a pertinência, viabilidade e eficácia. As avaliações 'durante' têm como objetivo básico fornecer informações sobre o andamento do programa com ponderação dos resultados alcançados até o momento. As avaliações ex post são as realizadas: dão informação sobre a execução, funcionamento e resultados (efeitos).

Segundo a procedência dos avaliadores, a avaliação pode ser externa, interna e mista. A avaliação externa tem como objetivo emitir conclusões e fazer recomendações; a interna, conhecer melhor o próprio trabalho e utilizar os resultados para melhorá-lo, enquanto as avaliações mistas têm a vantagem de possibilitar à equipe do projeto examinar o seu próprio trabalho, permitindo, também, conhecer o ponto de vista dos avaliadores externos, equilibrando fatores desfavoráveis e reforçando os favoráveis.

Quanto à função da avaliação, estas podem ser somativas e formativas. A formativa é uma avaliação de processos, pois se refere aos meios de execução de um programa e serve para retroalimentar o desenvolvimento das estratégias propostas inicialmente. A somativa refere-se à avaliação de resultados ou efeitos de um programa, auxiliando na tomada de decisão de manutenção, ou não, de um programa.

A classificação das avaliações, segundo os componentes do programa, que são objetos de avaliação, está longe da possibilidade de existir algum consenso entre os autores que se dedicam ao assunto. Nesse sentido, optouse, neste trabalho, por tomar como referência as classificações propostas por Ballart (1992), Aguilar e Ander-Egg (1994) e Figueiredo e Figueiredo (1996), pois entende-se que as divergências entre elas são apenas nas denominações dadas para a avaliação.

A avaliação do plano e conceituação do programa, como proposta por Aguilar e Ander-Egg (1994), avalia o modelo de intervenção social utilizado 
para resolver uma situação problema, julgando basicamente a pertinência e potencialidade de um programa. Para Ballart (1992), a avaliação da conceituação e desenho do programa deve propiciar informações sobre o problema a ser trabalhado, o processo de formulação do programa e analisar até que ponto o desenho do programa serve aos objetivos.

É consenso que, quando um programa é colocado em funcionamento, torna-se necessário avaliá-lo para que se tenha condição de reestruturá-lo em seu funcionamento. No entanto, essa avaliação pode ser global ou referir-se apenas a algum aspecto específico do processo de execução do programa.

É nesse sentido que a avaliação da instrumentação e seguimento do programa, como proposto por Aguilar e Ander-Egg (1994), e a avaliação da implementação de um programa, por Ballart (1992), são semelhantes. Esses tipos de avaliações compreendem aspectos como: a cobertura do programa; como o programa se operacionaliza; e os recursos humanos disponíveis e responsáveis pela operacionalização do programa.

As avaliações de processos, como proposta por Figueiredo e Figueiredo (1986), visam a aferir se o programa está sendo ou foi implantado conforme as diretrizes estabelecidas para a sua execução, ou se o produto atingirá, ou atingiu, as metas desejadas. Para esses autores existem distintos tipos de avaliação de processos: de metas (avaliação da eficácia objetiva); dos meios que podem ser de eficácia funcional, administrativa e contábil; e da eficiência (relação custo-benefício e custo-resultado).

A denominação dada por Aguilar e Ander-Egg (1994) de avaliação de eficiência ou rentabilidade econômica de um programa leva em conta o custo do programa em relação aos resultados obtidos. Para avaliar eficiência, estes autores propõem um tipo a mais de análise além das propostas por Ballart, Figueiredo e Figueiredo: a do custo-eficácia, também denominada como custo-efetividade, custo-benefício ou custo-utilidade.

A denominação de avaliação de eficácia ou efetividade, utilizada por Aguilar e Ander-Egg (1994), na verdade é uma avaliação de resultados, pois consiste basicamente em analisar até que ponto estão sendo alcançados os resultados previstos. Coincide, portanto, com a denominação de avaliação de impacto proposta por Figueiredo e Figueiredo (1986), e a denominação de avaliação de eficácia ou impacto do programa proposta por Ballart (1992). Esse tipo de avaliação consiste em demonstrar até que ponto as mudanças ocorridas são devidas à intervenção ou não.

Na América Latina, os mais recentes enfoques avaliativos tendem a fazer com que a avaliação torne-se parte do próprio trabalho, de um modo permanente e, não mais, apenas uma etapa de um ciclo de um projeto. Entretanto, esse enfoque traz consigo o desafio de superar a dicotomia ainda existente entre a utilização de métodos quantitativos e qualitativos. 
Os estudos avaliativos de políticas e programas sociais comportam a combinação de ambas metodologias, tendência de superação de enfoques dicotômicos. Sua natureza operativa faz com que se preocupe em subsidiar a gestão, o que a caracteriza como um tipo de pesquisa estratégica.

Tradicionalmente, têm-se utilizado os objetivos dos programas como critérios de avaliação, o que é pertinente quando os objetivos são produtos da negociação política e reflitam, em boa medida, os interesses dos envolvidos. A premissa teórica, que geralmente embasa o modelo clássico de avaliação por objetivos, é o desenho lógico da intervenção proposta. Nesses modelos de avaliação, os objetivos do programa são privilegiados, objetivam demonstrar que as mudanças nos indicadores selecionados são devidas à intervenção do programa (Ballart, 1992).

Entretanto, a avaliação por objetivos tem apresentado problemas, uma vez que os objetivos nem sempre estão claros, geralmente são abstratos, múltiplos e até mesmo contraditórios em algumas situações. Em certos casos, os programas podem ter efeitos não esperados que podem ser tão importantes como os expressos previamente, e, ainda, porque os atores dos programas tendem a definir objetivos mais modestos ou bem mais elevados do que os reais.

Ballart (1992), no estudo do caso do Plan Integral de Roquetes (PIR), ao caracterizar esse programa como de objetivos abrangentes, gerais e abertos, de forma pouco estruturada e em constante evolução, adotou uma aproximação ampla e levou em conta os reais interesses dos atores envolvidos. Utilizou metodologia intensiva em entrevistas e observações e perspectiva pluralista, aberta às opiniões dos atores envolvidos com o programa, dada à importância de reconhecer a diversidade de interesses em jogo e as suas múltiplas perspectivas na hora de processar um programa.

Essa perspectiva é interessante por permitir a reconstrução dos objetivos não claramente definidos anteriormente, e, ainda, conhecer o processo de negociação na formulação do programa, através de reconstituição dos reais interesses e objetivos dos atores participantes do programa de integração ensino-serviços.

\section{Metodologia}

Esta pesquisa caracterizou-se como um estudo de caso de um programa. Adotou-se a perspectiva utilizada por Ballart no estudo do caso do Plan Integral de Roquetes.

A seleção desse estudo se deu por ser, na época, a única experiência em curso entre a UFMT e os Serviços de Saúde de Mato Grosso, formalizada através de convênio e operacionalizada por tempo suficiente para apresen- 
tar resultados que poderiam ser analisados, bem como pela inexistência de estudos semelhantes de análise das diversas e distintas experiências de realização do Internato Rural Médico em parceria com os serviços de saúde desde o início de 1986.

Apesar de o Consórcio estar constituído por 11 municípios no ano de 2000, o estudo limitou-se a três (Sorriso, Nova Mutum e Lucas do Rio Verde), pois somente esses receberam alunos em todos os rodízios ocorridos naquele ano. Na definição do grupo de estudo, não se preocupou com rigor quantitativo, mas sim que cada ator fosse representativo e que detivesse uma imagem particular da cultura de sua instituição e do contexto de realização do programa.

Assim, o grupo de estudo foi constituído pelos gestores das instituições (GI) envolvidas e pelos agentes implementadores (AI) do programa. O critério de inclusão dos gestores da UFMT, dos municípios e do Consórcio foi terem exercido cargo de direção em suas respectivas instituições no ano de 2000, enquanto para o gestor da Secretaria de Estado de Saúde exigiu-se que tivesse sido o responsável pela condução do Programa de Integração, ou seja, esses atores deveriam ter sido os responsáveis em suas respectivas instituições pela coordenação, formulação e decisões políticas relacionadas ao programa. Os gestores selecionados foram: os secretários municipais de saúde, o secretário executivo e o presidente do Consórcio Intermunicipal de Saúde da Região do Teles Pires, o diretor e o chefe do departamento de Saúde Coletiva do Instituto de Saúde Coletiva (ISC), o diretor da Faculdade de Ciências Médicas e o coordenador de Recursos Humanos da Secretaria de Estado de Saúde de Mato Grosso.

A seleção dos agentes implementadores da UFMT e dos municípios pautou-se no fato de que os primeiros deveriam ser docentes-supervisores do Internato Rural Médico do ISC, enquanto os segundos deveriam ser os profissionais responsáveis pela execução das ações de saúde e acompanhassem diretamente as atividades desenvolvidas pelos alunos. Os agentes selecionados foram os cinco docentes do Internato Rural Médico e três preceptores dos municípios.

Como no ano de 2000 houve dois secretários de saúde no município de Lucas do Rio Verde, ambos foram entrevistados. Dos docentes do ISC, dois foram excluídos, tendo em vista um deles também desenvolver atividade laboral em um dos municípios, e o outro ter acompanhado as atividades de forma descontínua no ano selecionado. O secretário de Saúde de Nova Mutum e o presidente do Consórcio também não foram entrevistados, uma vez que o primeiro não foi localizado e o segundo não conseguiu compatibilizar sua agenda para a realização da entrevista.

Usou-se como fontes de dados o convênio $\mathrm{n}^{\circ} 024 / 98$, os termos aditivos e as entrevistas semi-estruturadas realizadas com os gestores e 
implementadores. Tais entrevistas ocorreram no período de abril a junho de 2002. Foram gravadas e, posteriormente, transcritas. Antecedeu a realização das entrevistas a anuência dos entrevistados em termo de consentimento livre e esclarecido.

A obtenção dos dados privilegiou as seguintes dimensões de análise: a) perspectivas das instituições envolvidas no programa; b) perfil sócioeconômico ocupacional dos gestores e agentes implementadores; c) negociações para a implantação do programa e participação das instituições; d) objetivos e expectativas dos atores em relação ao programa; e) importância atribuída pelos atores em relação às ações desenvolvidas pelos alunos; f) alcance, ou não, dos objetivos dos atores, a partir da realização do estágio; e g) dificuldades e facilidades na operacionalização do programa.

As atividades desenvolvidas foram avaliadas pelos atores, segundo o grau de importância para o alcance dos objetivos referidos, utilizando-se de uma escala valorativa. Os critérios adotados permitiram aos entrevistados atribuírem nota de 1 a 6 a cada item abordado, segundo os seguintes graus de importância: (1) nenhuma importância; (2) importância razoável; (3) importante; (4) fundamental; (5) a atividade não foi desenvolvida e (6) sem conhecimento para avaliar.

No tratamento dos dados, utilizou-se estatística descritiva às perguntas fechadas e categorização e tabulação das respostas às perguntas abertas. Realizou-se leitura sistemática das entrevistas, sendo seus resultados agrupados por instituição e por tipo de ator. Os objetivos reconstituídos foram categorizados em objetivos políticos, de integração institucional, acadêmicos e de organização de serviços, possibilitando assim destacar as opiniões mais freqüentes.

O projeto de pesquisa foi submetido ao Comitê de Ética em Pesquisa da Universidade Federal de Mato Grosso, tendo sido aprovado.

\section{Resultados}

O grupo de estudo foi constituído por 14 entrevistados, a saber: oito gestores institucionais (GI), sendo um GI da SES-MT, três da UFMT, um do Consórcio e três de municípios e seis agentes implementadores (AI), sendo três AI da UFMT e três de municípios. Entre os entrevistados, 12 tinham pós-graduação, sendo $75 \%$ especialistas, $16,7 \%$ mestres e $8,3 \%$ doutores.

Foram reconstituídos 47 objetivos, reagrupados em 20, tendo em vista a similaridade entre eles (Tabela 1). Desses objetivos, 15 foram citados por gestores e 13 por implementadores. Dos citados pelos gestores, $60 \%$ foram citados por um gestor, $26,7 \%$ por dois e $13,3 \%$ por quatro. Dos citados 


\section{pelos implementadores, $61,5 \%$ foram citados por um implementador, $15,4 \%$ por dois, $15,4 \%$ por três e $7,7 \%$ por quatro deles.}

Tabela 1

Objetivos consolidados do programa de integração ensino-serviços de saúde por gestores e implementadores

\begin{tabular}{|c|c|c|}
\hline Objetivos & Gestores $\left(n^{\circ}\right)$ & Implementadores $\left(\mathrm{n}^{\circ}\right)$ \\
\hline Buscar a integração da universidade com os serviços de saúde & 2 & - \\
\hline Fortalecer a implantação dos consórcios em Mato Grosso & 1 & 1 \\
\hline Fortalecer a política de saúde estadual & 1 & 1 \\
\hline Contribuir com a organização dos serviços de saúde municipais (Unidades & 4 & 2 \\
\hline Básicas de Saúde) & & \\
\hline Contribuir com a implantação e implementação dos serviços prestados pelo PSF & 2 & 2 \\
\hline $\begin{array}{l}\text { Contribuir para a formação de profissionais mais voltados para a prática } \\
\text { da saúde coletiva }\end{array}$ & 2 & 3 \\
\hline Implementar o atendimento médico por parte dos alunos & 2 & - \\
\hline Induzir os profissionais da rede básica a refletir acerca da própria atuação & 1 & - \\
\hline Promover a interiorização dos profissionais médicos formados na UFMT & 1 & 3 \\
\hline Apoiar e viabilizar a realização do Internato Rural Médico da UFMT & 1 & - \\
\hline Cumprir os objetivos acadêmicos de ensino aprendizagem & 4 & 4 \\
\hline Mudar as diretrizes curriculares do curso de medicina da UFMT & 1 & - \\
\hline Cumprir os trâmites burocráticos na garantia do convênio & 1 & - \\
\hline Ter campo formal para a realização do Internato Rural & 1 & 1 \\
\hline $\begin{array}{l}\text { Elaborar, conjuntamente universidade e serviços de saúde, propostas de } \\
\text { intervenção para os municípios }\end{array}$ & - & 1 \\
\hline Ter canal aberto com a universidade & - & 1 \\
\hline Ter assessoria qualificada por parte do corpo docente do ISC & - & 1 \\
\hline Utilizar os alunos como força de trabalho & - & 1 \\
\hline Incentivar a organização da atenção primária através da implementação do PSF & 1 & - \\
\hline Contribuir na reorientação do modelo de ensino atual do curso de medicina & - & 1 \\
\hline
\end{tabular}

Fonte: $\mathrm{O}$ autor. Pesquisa de campo. 
Diante da diversidade dos objetivos, estes foram reagrupados em quatro grandes áreas: política, que se referia à atuação política e à política de saúde propriamente dita; de integração institucional, que tinha como meta o fortalecimento da parceria/integração entre as instituições; acadêmica, que visava ao cumprimento ou reorganização das diretrizes curriculares do curso de medicina; de organização de serviços, que almejava o equacionamento dos problemas de organização dos serviços e assistência médica (Tabela 2).

Tabela 2

Distribuição dos objetivos do programa de integração ensino-serviços de saúde, segundo a classificação por área

\begin{tabular}{lrr}
\hline Classificação dos objetivos & $\mathrm{n}^{\circ}$ & $\%$ \\
\hline 1 - Políticos & 2 & 10
\end{tabular}

- Fortalecer a implantação dos consórcios no Estado

- Fortalecer a política estadual de saúde

- Buscar a integração da universidade com os serviços de saúde

- Ter canal aberto com a universidade

- Ter assessoria qualificada por parte do corpo docente do ISC

\section{3 - Acadêmicos}

- Contribuir para a formação de profissionais mais voltados para a prática da saúde coletiva

- Promover a interiorização dos profissionais médicos formados na UFMT

- Cumprir os objetivos acadêmicos de ensino-aprendizagem

- Mudar as diretrizes curriculares do curso de medicina da UFMT

- Ter campo formal para a realização do Internato Rural

- Contribuir na reorientação do modelo de ensino atual do curso de medicina

\section{4 - Organização de serviços}

- Contribuir para a organização dos serviços de saúde municipais (UBS)

- Contribuir com a implantação e implementação dos serviços prestados pelo PSF

- Implementar o atendimento médico por parte dos alunos

- Induzir os profissionais da rede básica a refletir acerca da própria atuação

- Apoiar e viabilizar a realização do Internato Rural Médico da UFMT

- Representar as prefeituras municipais junto a outras instituições

- Elaborar, conjuntamente universidade e serviços de saúde, propostas de intervenção

para os municípios

- Utilizar os alunos como força de trabalho

- Incentivar a organização da atenção primária, através da implementação do PSF

Fonte: $\mathrm{O}$ autor. Pesquisa de campo.

De acordo com os dados apresentados, 75\% dos objetivos eram relativos a demandas específicas das instituições executoras, ou seja, objetivos 
acadêmicos e de organização de serviços, e 15\% se referiam à integração entre a universidade e os serviços de saúde.

A classificação dos objetivos apresentados pelos gestores e implementadores nas quatro áreas demonstrou que, dos 15 objetivos citados pelos gestores, 6,7\% eram de integração institucional, 33,3\% acadêmicos e 46,7\% de organização de serviços. Dos 13 objetivos citados pelos implementadores, $15,4 \%$ eram políticos, 15,4\% de integração institucional, 38,4\% acadêmi$\cos$ e $30,8 \%$ de organização de serviços. Esses resultados mostraram o foco dos atores em questões específicas das suas instituições de origem, ficando a integração institucional em plano secundário.

A compilação dos 20 objetivos segundo instituição demonstrou que 11 foram citados pela UFMT, quatro pela SES-MT, 14 pelos municípios e um pelo Consórcio. Onze (55\%) dos objetivos foram citados por atores de uma das instituições e oito (40\%) foram coincidentes para atores de duas instituições. A interiorização dos profissionais médicos formados pela UFMT foi o único objetivo coincidente entre as instituições: universidade, secretaria de estado de saúde e municípios.

A classificação dos objetivos apresentados pelos atores, por área e analisados por instituições, está representada na Tabela 3. Observou-se que dos 11 objetivos citados pela universidade, 18,2\% foram políticos, 54,5\% acadêmicos e $27,3 \%$ de organização de serviços. Dos quatro citados pela SES-MT, 25\% eram objetivos de integração institucional, 25\% acadêmicos e $50 \%$ de organização de serviços. Dos objetivos citados pelos municípios, 14,3\% foram políticos, $21,4 \%$ de integração institucional, 21,4\% acadêmicos e $42,9 \%$ de organização de serviços. O único objetivo apresentado pelo Consórcio referia-se à organização de serviços.

Tabela 3

Classificação dos objetivos do programa de integração ensino-serviços de saúde por área, segundo instituições

\begin{tabular}{lcccccccc}
\hline Classificação dos objetivos & \multicolumn{2}{c}{ UFMT } & \multicolumn{3}{c}{ SES } & \multicolumn{2}{c}{ Municípios } & \multicolumn{2}{c}{ Consórcio } \\
$\left(\mathrm{n}^{\circ}\right)$ & $(\%)$ \\
\hline 1. Políticos de saúde & 2 & $(\%)$ & $\left(n^{\circ}\right)$ & $(\%)$ & $\left(n^{\circ}\right)$ & $(\%)$ & - \\
2. Integração institucional & - & - & 1 & 25 & 3 & 21,4 & - & - \\
3. Acadêmicos & 6 & 54,5 & 1 & 25 & 4 & 21,4 & - & - \\
4. Organização de serviços & 3 & 27,3 & 2 & 50 & 5 & 42,9 & 1 & 100 \\
\hline Total & 11 & 100 & 4 & 100 & 14 & 100 & 1 & 100
\end{tabular}

Fonte: $\mathrm{O}$ autor. Pesquisa de campo.

Ficou evidente o predomínio dos objetivos acadêmicos e de organização de serviços, em $81,8 \%, 75 \%$ e $64,3 \%$ para os atores da UFMT, SES e 
municípios, respectivamente. A universidade não apresentou nenhum objetivo de integração institucional e a secretaria nenhum objetivo político. O objetivo de buscar a integração da universidade com os serviços de saúde foi apresentado somente pela SES e por um dos municípios, demonstrando que a integração não poderia acontecer de forma satisfatória, uma vez que apenas dois gestores tinham em perspectiva a integração institucional. Percebeu-se que as instituições buscaram o alcance dos seus objetivos específicos, o que se entende ser importante em projetos dessa natureza, entretanto, ao se limitar a eles, elas deixaram de fortalecer a parceria e de potencializar o trabalho integrado.

As atividades desenvolvidas e o grau de importância atribuído pelos gestores e implementadores para o alcance dos objetivos reconstituídos estão demonstrados na Tabela 4.

A assessoria dos docentes do ISC aos municípios foi avaliada como importante e fundamental por $90 \%$ dos entrevistados e $100 \%$ dos implementadores.

Os estudos diagnósticos da situação de saúde e elaboração de perfis epidemiológicos foram considerados importantes e fundamentais por 85,7\% dos entrevistados. Segundo categoria de atores, essa atividade foi importante e fundamental para $87,5 \%$ dos gestores e $83,3 \%$ dos implementadores.

A implementação das atividades propostas nos planos municipais de saúde foi analisada como importante e fundamental por $77,8 \%$ dos entrevistados; $100 \%$ dos gestores avaliaram-na como importante; e $50 \%$ dos implementadores como de importância razoável. Percebe-se assim divergência entre os atores.

A implementação e análise de bancos de dados foram atividades importantes e fundamentais para $70 \%$ dos entrevistados e para $100 \%$ dos implementadores. Já para $40 \%$ dos gestores esta atividade foi considerada sem nenhuma importância.

Quanto ao planejamento e programação em saúde, houve dispersão entre os critérios avaliativos, em relação a sua relevância para o conjunto dos atores, entretanto $83,3 \%$ dos implementadores consideraram-na importante e fundamental.

Os treinamentos-capacitações às equipes do PSF foram importantes para $54,5 \%$ dos entrevistados, no entanto, destacados neste critério por $80 \%$ dos implementadores.

As atividades de educação em saúde e de atendimento médico às populações urbanas e rurais obtiveram avaliação dispersa entre os critérios, indicando a pouca relevância atribuída a essas atividades. Quanto ao atendimento às populações rurais, predominou avaliação de 'importância razoável' para todos os atores. 


\begin{tabular}{|c|c|c|c|c|c|c|c|c|c|c|c|c|}
\hline \multirow{3}{*}{ Atividades desenvolvidas } & \multicolumn{12}{|c|}{ Scores da avaliação } \\
\hline & \multicolumn{4}{|c|}{ Atores } & \multicolumn{4}{|c|}{ Gestores } & \multicolumn{4}{|c|}{ Implementadores } \\
\hline & $\begin{array}{l}1^{*} \\
(\%)\end{array}$ & $\begin{array}{c}2^{*} \\
(\%)\end{array}$ & $\begin{array}{l}3^{*} \\
(\%)\end{array}$ & $\begin{array}{l}4^{*} \\
(\%)\end{array}$ & $\begin{array}{c}1 \\
(\%)\end{array}$ & $\begin{array}{c}2 \\
(\%)\end{array}$ & $\begin{array}{c}3 \\
(\%)\end{array}$ & $\begin{array}{c}4 \\
(\%)\end{array}$ & $\begin{array}{c}1 \\
(\%)\end{array}$ & $\begin{array}{c}2 \\
(\%)\end{array}$ & $\begin{array}{c}3 \\
(\%)\end{array}$ & $\begin{array}{c}4 \\
(\%)\end{array}$ \\
\hline $\begin{array}{l}\text { Estudos diagnósticos da situação } \\
\text { de saúde e elaboração de perfis } \\
\text { epidemiológicos }\end{array}$ & 7,1 & 7,1 & 50 & 35,7 & 12,5 & - & 50 & 37,5 & - & 16,7 & 50 & 33,3 \\
\hline $\begin{array}{l}\text { Ações de planejamento e } \\
\text { programação em saúde }\end{array}$ & 14,3 & 21,4 & 28,6 & 35,7 & 25 & 25 & 12,5 & 37,5 & - & 16,7 & 50 & 33,3 \\
\hline $\begin{array}{l}\text { Implementação de atividades } \\
\text { propostas nos planos municipais } \\
\text { de saúde }\end{array}$ & - & 22,2 & 66,7 & 11,1 & - & - & 100 & - & - & 50 & 25 & 25 \\
\hline $\begin{array}{l}\text { Atendimento médico à população } \\
\text { urbana }\end{array}$ & - & 42,9 & 28,6 & 28,6 & - & 37,5 & 25 & 37,5 & - & 50 & 33,3 & 16,7 \\
\hline $\begin{array}{l}\text { Atendimento médico à população } \\
\text { rural }\end{array}$ & - & 57,1 & 21,4 & 21,4 & - & 50 & 12,5 & 37,5 & - & 66,7 & 33,3 & - \\
\hline $\begin{array}{l}\text { Treinamentos / capacitações às } \\
\text { equipes do PSF }\end{array}$ & 9,1 & 27,3 & 54,5 & 9,1 & 16,7 & 33,3 & 33,3 & 16,7 & - & 20 & 80 & - \\
\hline $\begin{array}{l}\text { Educação em saúde junto a escolas } \\
\text { e comunidades }\end{array}$ & 8,3 & 33,3 & 41,7 & 16,7 & 16,7 & 33,3 & 33,3 & 16,7 & - & 33,3 & 50 & 16,7 \\
\hline $\begin{array}{l}\text { Implantação e análise de bancos } \\
\text { de dados }\end{array}$ & 20 & 10 & 50 & 20 & 40 & 20 & 20 & 20 & - & - & 80 & 20 \\
\hline $\begin{array}{l}\text { Assessoria dos docentes do ISC } \\
\text { aos municípios }\end{array}$ & - & 9 & 45,5 & 45,5 & - & 16,7 & 66,7 & 16,7 & - & - & 20 & 80 \\
\hline
\end{tabular}

Os dados apresentados mostram que apenas a assessoria dos docentes e os estudos de diagnósticos foram relevantes para o conjunto dos atores. Observou-se considerável número de divergências entre os gestores e implementadores quanto à importância da implementação de atividades dos planos municipais, implantação e análise de bancos de dados, planejamento e programação em saúde e capacitações das equipes do PSF. Essas divergências, provavelmente relacionadas à posição diferenciada dos atores na hierarquia das instituições, atribuíam valores diferentes às mesmas ações. Tais diferenças podem vir a se constituir em resistências e conflitos. 
A freqüência de escolha dos critérios de 'nenhuma importância' ou 'importância razoável' provavelmente possa explicar-se na fala de um dos entrevistados, que destacou o aspecto burocrático de algumas ações do estágio.

Muitas ações orientadas pelos professores e realizadas pelos alunos se transformam em relatórios, que ficam perdidos em pilhas de papéis no ISC, significando que esses trabalhos estão sendo realizados apenas para dar trabalho aos alunos e não com uma finalidade de apontar soluções que tenham continuidade (GI da instituição A).

A importância atribuída às atividades desenvolvidas, segundo as instituições, está demonstrada na Tabela 5. Constatou-se que para os atores da secretaria de estado de saúdeas atividades consideradas importantes e fundamentais foram os estudos diagnósticos da situação de saúde e elaboração de perfis epidemiológicos, o atendimento médico à população urbana e rural e a implantação e análise de bancos de dados.

Segundo $80 \%$ dos atores da UFMT, a assessoria dos docentes do ISC aos municípios, os estudos diagnósticos da situação de saúde e elaboração de perfis epidemiológicos, as ações de planejamento e programação em saúde, a implementação de atividades propostas nos planos municipais de saúde e a implantação e análise de bancos de dados foram as atividades importantes e fundamentais para contribuir no alcance dos objetivos da instituição. Já os treinamentos-capacitações às equipes do PSF e as atividades de educação em saúde junto a escolas e comunidade não apresentaram consenso entre os entrevistados.

Os atores municipais priorizaram os treinamentos-capacitações às equipes do PSF, os estudos diagnósticos da situação de saúde e elaboração de perfis epidemiológicos, a implementação de atividades propostas nos planos municipais de saúde e a assessoria dos docentes do ISC aos municípios. Essas atividades foram avaliadas como importantes ou fundamentais por, pelo menos, $75 \%$ deles. Entre os atores municipais, as ações de planejamento e programação em saúde não apresentaram consenso quanto a sua relevância.

Para o gestor do Consórcio, as atividades consideradas importantes e fundamentais foram as ações de planejamento e programação em saúde; os atendimentos médicos a população urbana e rural e os estudos diagnósticos da situação de saúde e elaboração de perfis epidemiológicos. 
Avaliação das atividades desenvolvidas no programa de integração ensino-serviços de saúde, segundo instituição

\begin{tabular}{|c|c|c|c|c|c|c|c|c|c|c|c|c|c|c|c|c|}
\hline \multirow{3}{*}{ Atividades desenvolvidas } & \multicolumn{16}{|c|}{ Scores da avaliação } \\
\hline & \multicolumn{4}{|c|}{ SES } & \multicolumn{4}{|c|}{ UFMT } & \multicolumn{4}{|c|}{ Municípios } & \multicolumn{4}{|c|}{ Consórcio } \\
\hline & $\begin{array}{l}1 * \\
(\%)\end{array}$ & $\begin{array}{l}2^{*} \\
(\%)\end{array}$ & $\begin{array}{c}3^{*} \\
(\%)\end{array}$ & $\begin{array}{l}4^{*} \\
(\%)\end{array}$ & $\begin{array}{c}1 \\
(\%)\end{array}$ & $\begin{array}{c}2 \\
(\%)\end{array}$ & $\begin{array}{c}3 \\
(\%)\end{array}$ & $\begin{array}{c}4 \\
(\%)\end{array}$ & $\begin{array}{c}1 \\
(\%)\end{array}$ & $\begin{array}{c}2 \\
(\%)\end{array}$ & $\begin{array}{c}3 \\
(\%)\end{array}$ & $\begin{array}{c}4 \\
(\%)\end{array}$ & $\begin{array}{c}1 \\
(\%)\end{array}$ & $\begin{array}{c}2 \\
(\%)\end{array}$ & $\begin{array}{c}3 \\
(\%)\end{array}$ & $\begin{array}{c}4 \\
(\%)\end{array}$ \\
\hline $\begin{array}{l}\text { Estudos diagnósticos da situação } \\
\text { de saúde e elaboração de perfis } \\
\text { epidemiológicos }\end{array}$ & - & - & - & 100 & 16,7 & - & 33,3 & 50 & - & 16,7 & 50 & 33,3 & - & - & 100 & - \\
\hline $\begin{array}{l}\text { Ações de planejamento e } \\
\text { programação em saúde }\end{array}$ & - & 100 & - & - & 16,7 & - & 33,3 & 50 & 16,7 & 33,3 & 33,3 & 16,7 & - & - & - & 100 \\
\hline $\begin{array}{l}\text { implementação de atividades } \\
\text { propostas nos planos municipais } \\
\text { de saúde }\end{array}$ & - & - & - & - & - & 20 & 80 & - & - & 25 & 50 & 25 & - & - & - & - \\
\hline $\begin{array}{l}\text { Atendimento médico à população } \\
\text { urbana }\end{array}$ & - & - & 100 & - & - & 66,7 & 33,3 & - & - & 33,3 & 16,7 & 50 & - & - & - & 100 \\
\hline $\begin{array}{l}\text { Atendimento médico à população } \\
\text { rural }\end{array}$ & - & - & - & 100 & - & 50 & 33,3 & 16,7 & - & 66,7 & 16,7 & 16,7 & - & - & - & 100 \\
\hline $\begin{array}{l}\text { Treinamentos / capacitações às } \\
\text { equipes do PSF }\end{array}$ & - & 100 & - & - & 16,7 & 33,3 & 50 & - & - & - & 75 & 25 & - & - & - & - \\
\hline $\begin{array}{l}\text { Educação em saúde junto a escolas } \\
\text { e comunidades }\end{array}$ & - & - & - & - & 16,7 & 33,3 & 16,7 & 33,3 & - & 33,3 & 66,7 & - & - & - & - & - \\
\hline $\begin{array}{l}\text { Implantação e análise de bancos } \\
\text { de dados }\end{array}$ & - & - & 100 & - & 20 & - & 40 & 40 & - & 33,3 & 66,7 & - & 100 & - & - & - \\
\hline $\begin{array}{l}\text { Assessoria dos docentes do ISC } \\
\text { aos municípios }\end{array}$ & - & - & - & - & - & - & 33,3 & 66,7 & - & 25 & 25 & 50 & - & - & - & - \\
\hline
\end{tabular}

Fonte: $O$ autor. Pesquisa de campo.

Notas: (1) nenhuma importância $\quad$ (2) importância razoável $\quad$ (3) importante (4) fundamental

Percebe-se que somente a realização de estudos diagnósticos da situação de saúde foi relevante para todas as instituições. Os treinamentos das equipes do PSF foram importantes apenas para os municípios. A implantação das ações propostas nos planos municipais de saúde e a assessoria dos docentes do ISC aos municípios foram atividades convergentes entre a universidade e os municípios.

O fato de as ações de planejamento e programação em saúde e a implantação e análise de bancos de dados terem sido valorizadas pela UFMT, mas 
não tanto pelos municípios, provavelmente expresse a urgência dos atores municipais em solucionar problemas já conhecidos e priorizados.

Destaca-se que os atendimentos médicos efetuados pelos alunos, uma das expectativas dos prefeitos em relação ao estágio, não receberam dos atores municipais a mesma ênfase.

Apesar de a maioria das atividades desenvolvidas contribuir para o alcance dos objetivos de organização de serviços, foi possível perceber consideráveis divergências entre os atores.

A síntese do julgamento dos atores quanto ao alcance ou não dos objetivos com a integração entre o ensino e os serviços de saúde demonstrou que, para 40,5\% dos entrevistados, os objetivos foram alcançados; para $34 \%$ não foram alcançados; e para 25,5\%, alcançados parcialmente. Entre os gestores, $40 \%$ dos objetivos foram alcançados parcialmente e $32 \%$ classificados como alcançados. Entre os implementadores, 50\% dos objetivos foram alcançados e 45,5\% não alcançados.

A Tabela 6 mostra o alcance dos objetivos por instituição participante do programa. Para a UFMT, 45\% dos objetivos foram alcançados e $30 \%$ não alcançados. Para os municípios, 42,8\% dos objetivos não foram alcançados e, para a secretaria de estado de saúde, 50\% dos objetivos foram alcançados. O Consórcio foi a única instituição onde todos os objetivos foram alcançados.

Tabela 6

\begin{tabular}{|c|c|c|c|c|c|c|c|c|}
\hline \multicolumn{9}{|c|}{ Distribuição do alcance dos objetivos, segundo instituição } \\
\hline \multirow[t]{2}{*}{ Objetivos } & \multicolumn{2}{|c|}{ UFMT } & \multicolumn{2}{|c|}{ Municípios } & \multicolumn{2}{|c|}{ SES } & \multicolumn{2}{|c|}{ Consórcio } \\
\hline & $\left(n^{\circ}\right)$ & $(\%)$ & $\left(n^{\circ}\right)$ & $(\%)$ & $\left(n^{\circ}\right)$ & $(\%)$ & $\left(n^{\circ}\right)$ & (\%) \\
\hline Objetivos alcançados & 9 & 45 & 6 & 28,6 & 2 & 50 & 2 & 100 \\
\hline Objetivos alcançados parcialmente & 5 & 25 & 6 & 28,6 & 1 & 25 & - & - \\
\hline Objetivos não alcançados & 6 & 30 & 9 & 42,8 & 1 & 25 & - & - \\
\hline Total & 20 & 100 & 21 & 100 & 4 & 100 & 2 & 100 \\
\hline
\end{tabular}

Fonte: $\mathrm{O}$ autor.

Constatou-se que o percentual de alcance dos objetivos por categoria de atores foi baixo tanto para os gestores como para os agentes implementadores. Já por instituição, constatou-se que a universidade e a secretaria de estado de saúde foram as instituições que mais alcançaram os seus objetivos, ocorrendo situação inversa com os municípios.

Esses resultados, além de representar graus diferentes de satisfação entre os atores das instituições, demonstram que os atores municipais foram os que tiveram índices mais altos de insatisfação, o que leva a considerar 
que as atividades desenvolvidas não foram suficientes para possibilitar o alcance dos objetivos desses entrevistados.

Entre os pontos facilitadores e dificultadores identificados na realização do programa de integração, destaca-se que, para alguns atores, a simples formalização do convênio já estaria em parte possibilitando a concretização de alguns objetivos, como: a interiorização dos profissionais médicos formados na UFMT; ter campo formal para a realização do Internato Rural Médico; possuir canal aberto com a universidade; ter assessoria dos docentes do Instituto de Saúde Coletiva e o objetivo de apoiar a realização do internato. Esta linha de raciocínio mostra-se ingênua, porque, na verdade, só algumas questões operacionais estavam garantidas com a formalização do convênio, e não a viabilidade de estratégias e metas comuns, de diretrizes interinstitucionais que se consubstanciariam em ações nos serviços de saúde, respondendo, assim, às necessidades da população e aos interesses dos profissionais e alunos envolvidos.

A realização de um conjunto de ações, por si só, não foram capazes de sanar as lacunas surgidas pelo não estabelecimento de objetivos comuns às instituições e pela não-realização de avaliações periódicas e sistemáticas. Seguramente, estes fatos contribuíram para os altos índices de insatisfação detectados.

Em relação à não explicitação dos objetivos comuns para o programa, destacamos a fala de um dos entrevistados: “(...) como estes objetivos não estavam claros, então cada um se achava no bel prazer de decidir o que tinha que fazer; aí um fazia consulta, outro pesquisa, aí chegaram a uma confusão danada" [sic] (GI da instituição D).

Quanto à não realização de avaliações sistemáticas, que poderiam ter funcionado como mecanismo de redirecionamento das atividades, destacase o trecho de uma entrevista: “(...) por falta dessa comissão, cada convenente foi progressivamente fazendo aquilo que lhe era mais conveniente, desvirtuando parcialmente os objetivos originais" (GI da instituição D).

As reuniões realizadas ao término de cada rodízio do internato mostraram-se insuficientes para o acompanhamento e avaliação das atividades desenvolvidas. Apesar dessas dificuldades, a experiência foi validada por alguns atores.

(...) eu acho que é muito importante que aconteça isso (integração entre a universidade e os serviços de saúde), ela tem um peso importante no próprio serviço; ela criou uma oferta importante no serviço, só que criou expectativas que não foram cumpridas ou não deram conta, mas de qualquer jeito é muito válido, é importante pro serviço, pro aluno, pra própria gestão (GI da instituição B). 
O não funcionamento da comissão de acompanhamento e avaliação do programa que deveria ser composta por membros das instituições parceiras foi outro fator que contribuiu para o não-equacionamento dos conflitos e problemas que surgiram, bem como deixou de garantir o acompanhamento e avaliação das atividades e resultados parciais alcançados, comprometendo assim o realinhamento e adequações necessárias ao programa.

O acompanhamento das atividades desenvolvidas pelos alunos não ocorreu de forma homogênea, e tampouco com instrumento específico para esse fim. A curta permanência dos docentes junto aos alunos, quando das supervisões, foi outro fator muito destacado, uma vez que esse tempo não permitia aos docentes esclarecer todas as dúvidas dos alunos, gestores, preceptores municipais e da comunidade, bem como dar os encaminhamentos necessários para o bom andamento do internato e da integração.

Outro fator importante detectado foi que aproximadamente $70 \%$ dos implementadores municipais expressaram dificuldades em sensibilizar seus gestores quanto à importância de determinadas atividades e dos seus benefícios. Essa condição era agravada pelas demandas criadas pelos alunos, que, em muitos momentos, literalmente modificavam as rotinas das unidades de saúde, contribuindo com a insatisfação de profissionais das unidades de saúde.

Apesar da necessidade de apoio técnico aos serviços ter sido uma demanda forte e constante dos municípios, a assessoria dos docentes do Instituto de Saúde Coletiva não ocorreu como esperado, como pôde ser constatado em entrevista.

(...) eles (docentes) ajudaram, mas não vou dizer que foi $100 \%$, porque não foram assim, né? Os docentes vinham sempre muito rápido e a permanência dos mesmos no município se limitava à conversa com os alunos (GI da instituição C).

Os estudos diagnósticos foram considerados por implementadores e gestores municipais como obstáculo, uma vez que, 'ao invés de ajudar na solução' dos problemas conhecidos, 'criavam' novos. Avaliados ainda como insuficientes, foram programações de definição das atividades ao início dos rodízios dos estágios: "Em momento algum se fez programação de atividades e sim o atendimento das demandas da universidade e do município" (AI da instituição B).

Destaca-se como importante a limitação dos agentes implementadores da UFMT aos interesses acadêmicos e a solução de problemas operacionais diários, e o interesse dos gestores nas questões financeiras do convênio na relação com a SES-MT.

A existência de opiniões divergentes acerca das atividades dos alunos foi observada em parte das entrevistas. 
(...) eu entendi que não era para eles (alunos) virem aqui e nós aproveitarmos o serviço deles; eles informavam a gente através de relatórios e a partir daí a preceptoria começou a se beneficiar com o trabalho deles (GI da instituição C).

(...) o objetivo que predominava era o do aluno, pois os alunos vinham buscar primeiro para eles e alguns queriam buscar só para si (AI da instituição C).

(...) eu queria que os alunos ficassem atendendo o dia inteiro (GI da instituição D).

As opiniões divergentes sugerem a necessidade de se estabelecer referência teórica mais ampla e consistente que dê significado às ações dos atores, além dos objetivos institucionais específicos. A compreensão comum da integração entre as instituições poderia aproximar questões divergentes entre a Saúde Coletiva e a clínica, superando a falsa dicotomia entre o individual e o coletivo, expressa por um dos entrevistados:

Academicamente falamos em interdisciplinaridade, em integração e extensão, porém existe um imenso distanciamento entre o Instituto de Saúde Coletiva e a Faculdade de Ciências Médicas, e, conseqüentemente, há também um imenso distanciamento entre as ações didáticas realizadas pelos professores do ISC para o curso médico (não só do internato) e o Colegiado de Curso de Medicina. O Internato Rural passa a ser visto como um Internato da Saúde Coletiva e não do Curso (GI da instituição A).

Apesar das dificuldades, aspectos positivos foram destacados, a saber: a ampliação das equipes do Programa de Saúde da Família nos municípios e o aumento da presença de profissionais médicos egressos da UFMT no interior do estado.

Como o convênio não apresentou objetivos para a integração, e sim a diretriz de estabelecer condições para a realização de trabalho integrado de saúde entre as partes conventes, através do estágio e interiorização em Saúde da Família, esta causou incerteza no momento inicial da pesquisa, uma vez que, por um lado, ela continha a idéia de desenvolvimento de ações globais e de conjunto sobre a totalidade dos problemas das instituições envolvidas e, por outro, o caráter geral e amplo nos referenciou a idéia de intervenção integrada, enquanto forma de organizar os recursos das distintas instituições de acordo com uma estratégia e direção compartilhada.

Ao final, constatou-se que, em nenhum momento, os entendimentos apresentados aqui se concretizaram, ou seja, as ações desenvolvidas foram específicas, nem sempre integradas com estratégias comuns e compartilhadas e tampouco estiveram relacionadas à totalidade dos problemas das instituições envolvidas. 


\section{Considerações finais}

As mudanças que vêm sendo implementadas na organização do trabalho no interior do sistema de saúde brasileiro têm apontado para a necessidade de formar profissionais com perfil adequado às necessidades sociais, implicando, assim, que tenham habilidades de aprender a aprender, trabalhar em equipe, comunicar-se, ter agilidade frente às situações que se apresentam e ter capacidade propositiva. Nesse sentido, as iniciativas de integração entre os centros formadores e os serviços de saúde e as diretrizes curriculares nacionais do curso de graduação em medicina têm pautado suas atividades no sentido de desenvolver essas capacidades.

A análise do programa desenvolvido pela UFMT, SES-MT e o Consórcio revelou ambigüidade quanto à formulação, às características do programa, que em determinados momentos assumia feições de Integração Docente Assistencial (IDA) e, em outros, de União Ensino Serviços de Saúde (UNI), mas em nenhum momento a visão da Rede Unida.

Quanto ao processo de negociação para implantação do programa, constatou-se o interesse formal de implementá-lo, entretanto privilegiaram-se questões operacionais, sem o estabelecimento de um plano de trabalho conjunto que atendesse ao interesse das instituições e da própria integração.

Em relação aos objetivos dos atores relacionados ao programa, constatou-se que aproximadamente $60 \%$ dos objetivos reconstituídos não foram coincidentes entre as categorias de atores (gestores e implementadores), entre as instituições e, até mesmo, entre uma mesma categoria de atores. Dos objetivos reconstituídos, 55\% foram referidos por uma única instituição.

O agrupamento dos objetivos por área mostrou que $45 \%$ dos entrevistados formularam objetivos relacionados à organização dos serviços e 30\% relacionados a questões acadêmicas. Entre os gestores $(46,7 \%)$ predominaram os objetivos de organização de serviços e entre os implementadores $(38,4 \%)$, os acadêmicos. Entre as instituições, predominaram os objetivos acadêmicos, na UFMT e na secretaria de estado de saúde, Consórcio e municípios, os de organização de serviços.

Assim, pode-se dizer que houve baixa convergência entre os objetivos, e as instituições visaram muito mais concretizar seus objetivos específicos e não construir um programa embasado teoricamente no conceito de integração entre ensino e serviços de saúde, negociada entre elas.

Das atividades desenvolvidas, constatou-se, em algumas delas, elevado grau de discordância entre as instituições, entre os atores de uma mesma categoria e até mesmo entre os atores de uma mesma instituição, quanto à importância das mesmas para o alcance dos objetivos. Constatou-se, ainda, que alguns objetivos nem sequer tiveram atividades desenvolvidas que possibilitassem alcançá-los. 
Quanto ao alcance ou não dos objetivos, tanto por categoria de atores como por instituição, estes foram pouco alcançados, ou seja, $32 \%$ dos objetivos dos gestores e $50 \%$ dos implementadores foram alcançados, enquanto $45 \%$ dos objetivos da universidade e $50 \%$ dos objetivos da SES foram alcançados, reforçando o entendimento de que as divergências quanto à importância das atividades desenvolvidas refletiram diretamente no alcance dos objetivos.

Apesar de nenhum dos gestores ter sido contrário à integração, o envolvimento de alguns não ocorreu de maneira a contribuir efetivamente para a concretização da integração. Entendemos que essa falta de envolvimento se deu até mesmo por falta de mais discussões e informações acerca da proposta de integração.

Os gestores não utilizaram a autonomia e autoridade instituída pelo cargo no desempenho da função, no sentido de garantir todo o apoio logístico necessário para o bom desenvolvimento das atividades.

Das características assumidas pelo programa de integração estudado, podemos destacar que:

- na universidade, a integração ocorreu dentro de departamentos isolados e não no conjunto dos departamentos da Faculdade de Ciências Médicas, Faculdade de Enfermagem e Nutrição e Instituto de Saúde Coletiva;

- a integração, como se deu, favoreceu a dicotomia de médico generalista versus médico especialista;

- apesar de terem sido criados novos cenários de ensino além do hospital, não se criaram metodologias de ensino adequadas a esses cenários;

- a exposição dos alunos a esses novos cenários ocorreu de forma tardia;

- o sistema de saúde onde os alunos foram inseridos não foi organizado racional e previamente para que pudesse propiciar vivência aos estudantes do que se passa nos diversos níveis de atenção, contribuindo para a visão fragmentada da atenção primária à saúde por parte dos alunos;

- foram enfatizadas somente atividades e o ensino na atenção básica;

- elegeram-se, em sua grande maioria, comunidades marginalizadas e carentes, estimulando nos alunos a percepção de que a saúde pública é algo para populações carentes;

- a comunidade beneficiada foi passivo-receptiva e usada como objeto de estudo; e

- a responsabilidade e gestão da integração não foi compartilhada pelas instituições envolvidas.

A formalização da integração através de convênio pode ser uma estratégia suficiente para o alcance dos objetivos das instituições envolvidas, para a realização de estágios em saúde em municípios do interior do estado. Pode ainda ser um instrumento viável para a construção de relações cooperativas que contribuam na melhoria dos serviços de saúde e na formação médica em 
Mato Grosso, desde que existam princípios e objetivos comuns pactuados entre as instituições envolvidas.

Como não foi possível utilizar os objetivos do programa como critério de avaliação, tendo em vista não estarem definidos quando do início do programa, entendeu-se que se faz necessário, mesmo que tardiamente, estabelecer um referencial teórico sobre integração ensino-serviços de saúde que paute os objetivos, metas e ações, e a definição formal, coletiva e consensual: dos objetivos da integração; da situação esperada (metas); das atividades que serão desenvolvidas; das responsabilidades, atribuições e competências das instituições com a integração; de como se dará o monitoramento do programa; de quais os indicadores de processo e de resultado que serão utilizados para a avaliação da integração; e de como se buscará a sustentabilidade política da integração.

Por fim, entendeu-se que a definição prévia desses pontos contribuiria fundamentalmente para permitir a integração entre um centro formador e os serviços de saúde, garantindo o estabelecimento de relação entre as instituições que colaborassem para a reestruturação do modelo de ensino e da atenção à saúde.

\section{Notas}

${ }^{1}$ Docente do Instituto Brasileiro de Pós-Graduação e Extensão (IBPEX), Cuiabá, Mato Grosso, Brasil. Mestre em Saúde e Ambiente pela Universidade Federal de Mato Grosso (UFMT).<bertulio@terra.com.br>

Correspondência: Avenida Portugal, 30, Jardim Tropical, Cuiabá, Mato Grosso, Brasil, CEP 78.065-145.

2 Professora adjunta do Instituto de Saúde Coletiva da Universidade Federal de Mato Grosso (ISC/UFMT), Cuiabá, Mato Grosso, Brasil. Doutora em Saúde Coletiva pela Universidade Estadual de Campinas (Unicamp). <angsp@terra.com.br>

3 A Integração Docente-Assistencial (IDA) foi definida como união de esforços em um processo de crescente articulação entre instituições de educação e serviços de saúde, adequados às reais necessidades da população, à produção de conhecimento e à formação de recursos humanos necessários a um determinado contexto da prática de serviços de saúde e de ensino (Opas, MS, MEC, 1979).

4 "Processo de ensino pode ser definido como o conjunto de momentos sucessivos que envolvem atividades, meios e objetos de ensino pelos quais passa o estudante até transformar-se em médico" (Almeida, 1999a, p. 6). 
5 “Relações de ensino são as conexões ou vínculos que se estabelecem entre as pessoas participantes do processo de produção de médicos e são a resultante do papel que estes indivíduos desempenham no ensino médico" (Almeida, 1999a, p. 6).

6 Este artigo é parte da dissertação de mestrado defendida junto ao Instituto de Saúde Coletiva da Universidade Federal de Mato Grosso, em dezembro de 2003.

\section{Referências}

AGUILAR, Maria José; ANDER-EGG, Ezequiel. Avaliação de serviços e programas sociais. Petrópolis: Vozes, 1994. p. 17-57.

ALMEIDA, Marcio José. Educação médica e saúde: possibilidade de mudança. Rio de Janeiro: Editora da UEL e Abem, 1999a.

A educação dos profissionais de saúde na América Latina: teoria e prática de um movimento de mudança. São Paulo: Editora da UEL, Hucitec e Lugar Editorial S.A., tomo 1 e 2, 1999b.

BALLART, Xavier. Como evaluar programas y servicios públicos?: aproximación sistemática y estudios de caso. Madrid: Ministério para las Administraciones Publicas. 1992, p. 37-49.

BELACIANO, Mourad Ibrahim. O SUS deve aceitar este desafio: elaborar proposições para a formação e capacitação de recursos humanos em saúde. Divulgação em Saúde para Debate, n. 12 , p. 29-33, 1996.

DENIS, Jean-Louis; CHAMPAGNE, François. Análise da implantação. In: HARTZ, Zulmira M. de Araújo (Org.). Avaliação em saúde: dos modelos conceituais à prática na análise da implantação de programas. Rio de Janeiro: Editora Fiocruz, 2000, p. 49-87.

FEUERWERKER, Laura C. Macruz. Mudanças na educação médica e residência médica no Brasil. São Paulo: Hucitec/Rede Unida, 1998.
FIGUEIREDO, Argelina M. Cheibub; FIGUEIREDO, Marcus Faria. A avaliação política e avaliação de política. São Paulo. Análise e conjuntura, v. 1, n. 3, set.-dez., 1986.

KISIL, Marcos. Uma estratégia para a reforma sanitária: a iniciativa UNI. Rio de Janeiro, Divulgação em Saúde para Debate, n. 12, p. 5-14, 1996.

LAMPERT, Jadete Barbosa. Tendência de mudanças na formação médica no Brasil. Tese de doutorado. Escola Nacional de Saúde Pública, Fiocruz. Rio de Janeiro, 2002, p. 238.

MACHADO, José Lúcio Martins Machado. Fundação UNI-Botucatu: a construção de um novo paradigma na formação de profissionais da saúde. 1999. Disponível em: $<$ http://proex.reitoria.unesp.br/congressos/ Congressos/1_Congresso/Sa_de_e_Qualida de_de_Vida/Trabalho04.htm>. Acesso em: 13 jun. 2008.

MARSIGLIA, Regina Giffoni. Relação ensino/serviços: dez anos de integração docente assistencial (IDA) no Brasil. São Paulo: Hucitec, 1995.

MARTINIC, Sergio. Evaluación de proyectos. conceptos y herramientas para el aprendizaje. México: Comexani-Cejuv, 1997.

MINISTÉRIO DA EDUCAÇÃO. Conselho Nacional de Educação. Câmara de Educação 
Superior, 2001. Diretrizes Curriculares Nacionais do Curso de Graduação em Medicina. [resolução on-line] Disponível em: $<$ www.mec.gov.br/cne/ftp/CES/CES04.doc $>$ Acesso em: 14 ago. 2002.

OPAS/MS/MEC. Programa geral de desenvolvimento de recursos humanos para a saúde no Brasil. Brasília, 1979.
RODRIGUES, Maria Izabel. Tendências de la educación médica en América Latina en los últimos quince años. Las Profesiones en México, Medicina. México, n. 3, 1990.

YIN, Robert. Estudo de caso: planejamento e métodos. 2. ed. Porto Alegre: Bookman, 2001. p. 32.

Recebido em 16/04/2008

Aprovado em 29/06/2008 